\title{
Stopping power of large homonuclear clusters: Influence of cluster structure
}

\author{
M. Vicanek, ${ }^{*}$ I. Abril, N. R. Arista, ${ }^{\dagger}$ and A. Gras-Marti \\ Departament de Física Aplicada, Universitat d'Alacant, Apartat 99, E-03080 Alacant, Spain
}

(Received 6 April 1992)

\begin{abstract}
A theoretical investigation based on the linear dielectric formalism is presented of the stopping of large clusters in metals at velocities above the Fermi velocity. Calculations are carried out for hydrogen clusters of 4, 13, and $100 \mathrm{H}_{2}$ molecules stopping in aluminum. Emphasis is placed on the influence of the internal cluster structure, which enters in the present description via the pair-correlation function for the relative positions of nuclei. The target properties are modeled by Lindhard's dielectric function for a free-electron gas. Interference effects with respect to both collective and individual excitations are quantified separately for intra- and intermolecular terms, and are evaluated as a function of cluster velocity. Effects of short-range order, in particular the importance of an exclusion volume around each molecule, are clearly visible. The results indicate, however, that beyond the nearest neighbor the detailed molecular arrangement has only little influence on the stopping power for the clusters studied. The partitioning of contributions due to close and distant collisions, as it is known for the stopping of point charges, is found to be substantially different in the case of cluster projectiles. The effect of Coulomb repulsion is commented upon.
\end{abstract}

PACS number(s): 34.50.Bw, 36.40. $+\mathrm{d}$

\section{INTRODUCTION}

Most of our knowledge about the interaction processes that govern the behavior of swift ions in solids derives from experiments using beams of single atomic particles. In this case, the separation between the beam particles is microscopically so large that the solid is back to the equilibrium state when each new particle enters the medium, and so there is no interference in the response of the medium to different beam particles.

In contrast, when ionized cluster beams are used, the distance between the particles in a given cluster is similar to the interatomic distances in a solid, and therefore the cluster components will interact collectively. This can involve simultaneous interactions between several particles in the cluster and the solid. Hence, the use of cluster beams of molecular or atomic ions provides a new tool to investigate dynamical interaction processes in matter [1].

Most of the studies along this line considered the case of small clusters containing a few atomic ions [1-11]. In particular, earlier attention was paid to modifications in the energy loss of the cluster [2-7], to the Coulomb explosion of molecular ions [8,9], and to studies of spatial patterns to distinguish different molecular structures through cluster-beam-foil experiment analyses $[10,11]$.

More recently $[12,13]$, experimental techniques have been developed that allow the study of large molecular clusters containing up to several thousand molecules. This opens a field for new studies of cluster-solid interactions, where even the main processes are still not well understood; in particular, recent claims point at the possibility of using large molecular clusters to induce fusion processes in solids [14], an area that is currently under discussion $[14,15]$.

Furthermore, we should mention the growing field of ion-beam applications in fusion research, where energetic ion beams are used as drivers in inertial-confinement fusion experiments $[16,17]$. The possible use of ionizedcluster beams, which can provide higher concentrations of energy inside the target, is an alternative that becomes of particular interest. In a recent paper Deutsch [18] discusses experimental and theoretical aspects of the application of relatively large ionized cluster beams in particle-driven inertial confinement fusion.

Basic research is being pursued in the field of largecluster interaction with solid targets both theoretically [19] and experimentally. One recent experiment [20] was performed with hydrogen clusters $\mathrm{H}_{n}{ }^{+}$up to $n=25$. The authors measured the energy loss of clusters penetrating carbon foils at energies up to $120 \mathrm{keV}$ per proton.

In this work we consider the cluster stopping power (i.e., the energy loss per unit pathlength) in a solid due to electronic excitations, for a range of cluster velocities starting from the Fermi velocity $v_{F}$ of the solid-target electrons. We consider homonuclear clusters, and the electron gas model is used to account for the vicinage effects in both collective and individual electron excitations (i.e., the so-called electronic stopping power). By excluding clusters of low velocity we can ignore complications due to nonlinearity in the screening of the cluster charge [21], and also the effects of nuclear interaction on the energy loss, since electronic stopping is the dominant mechanism in the velocity range investigated here $[22,23]$. In particular, we analyze the influence of the cluster structure on the cluster energy loss, through a vicinage function that depends on the pair-correlation function and takes care of the collective interference among the particles in the cluster. Emphasis will be placed on the new field of stopping of large clusters.

In the next section we introduce the cluster stoppingpower model according to the linear dielectric formalism. In Sec. III we analyze various descriptions of cluster 
structure in terms of different assumptions on the paircorrelation function of hydrogen molecular clusters. Calculations of cluster stopping power are presented in Sec. IV, where the results of high-velocity approximations and exact numerical integrations for the full velocity range are shown. Section V summarizes the conclusions of this work.

\section{BASIC MODEL}

Consider a cluster consisting of $N$ atoms with relative positions $\mathbf{r}_{i j}$, each atom having a charge $Z$, and moving with velocity $\mathbf{v}$ through a stopping medium. A general expression for the stopping power $S_{\mathrm{cl}}$ of such a cluster is given by $[4,21,24]$

$$
S_{\mathrm{cl}}=Z^{2}\left[N+\sum_{i \neq j=1}^{N} I\left(r_{i j}, \theta_{i j}\right)\right] S_{p},
$$

where $S_{p}$ and $I(r, \theta)$ are, respectively, the proton stopping power and the interference function defined below. We use atomic units, $e=\hbar=m=1$, throughout this paper. Equation (1) takes into account the contribution of each individual particle to the stopping power as well as its interference effects with other particles in the cluster.

The electromagnetic response of the medium to an external perturbation is described by the dielectric formalism in terms of the target dielectric function $\epsilon(k, \omega)$. Thus, the proton stopping power is

$$
S_{p}=\frac{2}{\pi v^{2}} \int_{0}^{\infty} \frac{d k}{k} \int_{0}^{k v} d \omega \omega \operatorname{Im}\left(-\frac{1}{\epsilon(k, \omega)}\right),
$$

where $\operatorname{Im}(z)$ denotes the imaginary part of $z$. The interference function becomes

$$
\begin{aligned}
I(r, \theta)=\frac{1}{2 \pi^{2} v S_{p}} \int & d^{3} k \frac{\mathbf{k} \cdot \mathbf{v}}{k^{2}} \operatorname{Im}\left(-\frac{1}{\epsilon(k, \omega)}\right) \\
& \times \cos (\mathbf{k} \cdot \mathbf{r}),
\end{aligned}
$$

where the frequency $\omega$ is evaluated at $\omega=\mathbf{k} \cdot \mathbf{v}$. The angle between the velocity $\mathbf{v}$ and the vector $\mathbf{r}$ is denoted by $\theta$.

For random cluster orientation, Eq. (1) may be averaged over all orientations to give

$$
S_{\mathrm{cl}}=N Z^{2}\left[1+n \int d^{3} r g_{\mathrm{cl}}(r) I(r)\right] S_{p} .
$$

Here, $n$ stands for the number density of atoms in the cluster, while $g_{\mathrm{cl}}$ denotes the pair-correlation function for cluster atoms, i.e., $n g_{\mathrm{cl}}(r) d^{3} r$ is the average number of atoms found in a volume element $d^{3} r$ at distance $r$ from a randomly selected atom in the cluster. $I(r)$ denotes the angular average of the interference function $I(r, \theta)$,

$$
\begin{aligned}
I(r)=\frac{2}{\pi v^{2} S_{p}} \int_{0}^{\infty} & \frac{d k}{k} \frac{\sin (k r)}{k r} \\
& \times \int_{0}^{k v} d \omega \omega \operatorname{Im}\left[-\frac{1}{\epsilon(k, \omega)}\right) .
\end{aligned}
$$

Rearranging terms, Eq. (4) may be written in a condensed form,
$S_{\mathrm{cl}}=N Z^{2} \frac{2}{\pi v^{2}} \int_{0}^{\infty} \frac{d k}{k} F(k) \int_{0}^{k v} d \omega \omega \operatorname{Im}\left(-\frac{1}{\epsilon(k, \omega)}\right)$,

where the function $F(k)$ represents the structure factor [25] for the cluster,

$$
F(k)=1+n \int d^{3} r g_{\mathrm{cl}}(r) \frac{\sin (k r)}{k r} .
$$

As noted above, Eq. (6) is valid for random cluster orientation; the corresponding expression for oriented clusters is available $[21,24]$ but will not be used here since we concentrate on large clusters where, as discussed in Ref. [21], orientational effects are expected to be small.

Equation (6) can be applied to two limiting cases, the independent-particle limit and the united-atom limit. The first case may be realized by making all internuclear distances so large that the term $\sin (k r) /(k r)$ in Eq. (7) changes sign frequently and the net contribution from the interference term becomes very small. In this case, one obtains

$$
S_{\mathrm{cl}} \cong N Z^{2} S_{p}
$$

The united-atom limit, on the other hand, is obtained when internuclear distances may be considered to be small compared to the relevant excitation wavelengths; hence, one may replace the term $\sin (k r) /(k r)$ in Eq. (7) by unity, and the cluster stopping power becomes

$$
S_{\mathrm{cl}} \cong(N Z)^{2} S_{p}
$$

i.e., the stopping power of a pointlike particle with charge $N Z$. Here we have made use of the normalization condition,

$$
N=1+n \int d^{3} r g_{\mathrm{cl}}(r)=F(k=0) .
$$

The content of Eq. (9) should be understood merely as a formal result, since if all components of the cluster were contracted into one point charge, this would result in a strong perturbation of the target electrons and hence the assumption of a linear response of the medium would no longer be valid.

It may be noted that although Eqs. (8) and (9) are limiting cases with respect to the cluster size, they are not $e x$ treme cases with respect to the stopping power. For destructive interference the cluster stopping power may still be smaller than the independent-particle limit given by Eq. (8).

\section{CLUSTER STRUCTURE}

In order to calculate the cluster stopping power, one needs to specify the atomic pair-correlation function $g_{\mathrm{cl}}(r)$ for the cluster. This function contains information on the short- and, if present, long-range order in the cluster. In particular, the size and shape of the cluster also enter since $g_{\mathrm{cl}}(r)$ must vanish for separations exceeding the cluster size.

In the following, we shall investigate the stopping power of clusters of hydrogen molecules. This is the sim- 
plest theoretical case, and, as discussed in the Introduction, is also amenable to experimental study of cluster energy losses in solids.

From the experimental point of view, not much detail is known about the arrangement of molecules in hydrogen cluster beams. Ground-state configuration calculations are available [26] for small neutral $\left(\mathrm{H}_{2}\right)_{n}$ clusters. Moreover, claims exist [27] that singly ionized clusters typically appear as one $\mathrm{H}_{3}{ }^{+}$ionic core surrounded by $\mathrm{H}_{2}$ molecules. On the other hand, theoretical calculations [28] for alkali-metal clusters indicate that the lattice structure of a static cluster need not necessarily coincide with the bulk structure of the respective material, even for quite large clusters. It is one of the major points of the present investigation to assess to what extent the details of the molecular arrangement affect the stopping of the cluster.

Any reasonable model for the pair-correlation function of a hydrogen cluster has to take into account its molecular structure. A possible concept in this regard consists in dividing up the correlation function into an intra- and an intermolecular term

$$
g_{\mathrm{cl}}(r)=g_{\text {intra }}(r)+g_{\text {inter }}(r)
$$

corresponding to correlations in atomic positions within a molecular component of the cluster (intra), or correlations involving atoms belonging to different molecules (inter). The intramolecular term is represented by a sharp peak at the intramolecular separation $r_{\mathrm{mol}}=1.4$ a.u. (this value holds for a neutral molecule; the respective value for $\mathrm{H}_{2}{ }^{+}$is much larger, 2.44 a.u.),

$$
n g_{\text {intra }}(r)=\frac{\delta\left(r-r_{\text {mol }}\right)}{4 \pi r_{\text {mol }}^{2}} .
$$

The intermolecular term, on the other hand, contains information about the arrangement of molecules and obviously, about the size of the cluster. In view of the uncertainties regarding the structure of real, hot cluster ions, as they are used in beam experiments, we consider here two idealized models for the spatial arrangement of the molecules, which might be viewed as limiting cases of complete randomness and perfect order.

\section{A. Random spherical clusters}

Let the cluster consist of a single-element medium with atomic density $n$. By cutting out from this medium a sphere with radius $r_{\mathrm{cl}}$, one obtains a cluster with an average number of $N$ atoms,

$$
N=n \frac{4 \pi}{3} r_{\mathrm{cl}}^{3} \text {. }
$$

In this random model, the atoms form diatomic molecules, and these molecules are located at random positions within the cluster; yet no two atoms can approach each other closer than a minimum distance $r_{\mathrm{ex}}$, as for instance in a gas of hard spheres. The intermolecular pair correlation of atoms $g_{\text {inter }}(r)$ in such a cluster is zero for $r<r_{\mathrm{ex}}$, and is related, for $r \geq r_{\mathrm{ex}}$, to the probability $p$ of finding two atoms both within the sphere, at separation $r$,

$$
\begin{aligned}
p\left(r, r_{\mathrm{cl}}\right)=\left(\frac{4 \pi}{3} r_{\mathrm{cl}}^{3}\right)^{-1} \int & d^{3} r_{1} \\
& \times \int d^{3} r_{2} \frac{\delta\left(\left|\mathbf{r}_{1}-\mathbf{r}_{2}\right|-r\right)}{4 \pi r^{2}},
\end{aligned}
$$

where both integrations extend over the cluster volume. This amounts to calculating the overlap of two spheres of radius $r_{\mathrm{cl}}$ with their centers separated by $r$, giving

$$
p\left(r, r_{\mathrm{cl}}\right)=1-\frac{3}{2}\left[\frac{r}{2 r_{\mathrm{cl}}}\right]+\frac{1}{2}\left[\frac{r}{2 r_{\mathrm{cl}}}\right]^{3}
$$

for $r \leq 2 r_{\mathrm{cl}}$, and zero otherwise. The intermolecular part of the pair-correlation function for a random cluster is then given by

$$
g_{\text {inter }}(r)=C \Theta\left(r-r_{\mathrm{ex}}\right) p\left(r, r_{\mathrm{cl}}\right),
$$

with $\Theta(x)$ denoting the unit step function. The normalization constant

$$
C=\frac{N-2}{N}\left(1-8 x^{3}+9 x^{4}-2 x^{6}\right)^{-1}, x=\frac{r_{\mathrm{ex}}}{2 r_{\mathrm{cl}}}
$$

has been introduced here to conserve the number of atoms in the cluster, Eq. (10), hence to ensure compatibility with the united-atom limit Eq. (9). An example of the correlation function for a hydrogen cluster of 13 molecules is discussed in the next section.

\section{B. Ordered clusters}

In solid parahydrogen $\mathrm{H}_{2}$ molecules form a hexagonal close-packed lattice $[29,30]$, with no particular orientation of the molecular axes. In the ordered-cluster model we assume that hydrogen clusters will retain this structure. Two clusters of particularly high symmetry and low configurational energy are the tetrahedron, consisting of four $\mathrm{H}_{2}$ molecules, and one consisting of $13 \mathrm{H}_{2}$ molecules, shown in Fig. 1. These will be taken here to study effects of cluster structure on stopping.

The intermolecular pair-correlation function $g_{\text {inter }}$ for a

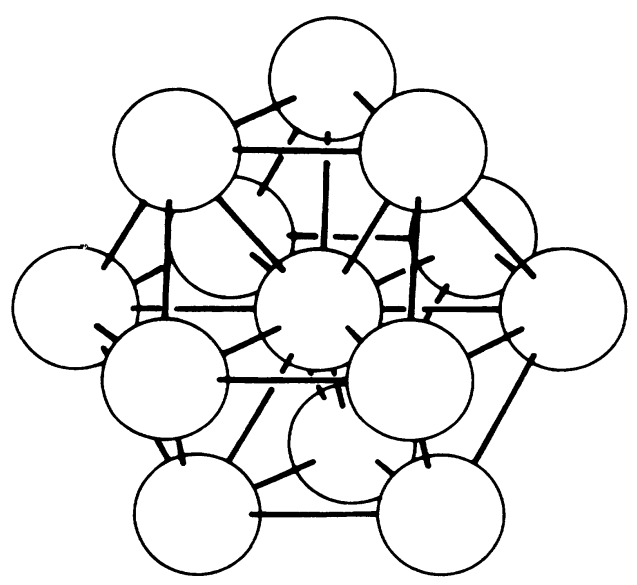

FIG. 1. Arrangement of 13 molecules in a cluster with hcp structure. 
TABLE I. Distribution of distances between lattice points (centers of $\mathrm{H}_{2}$ molecules) for the ordered cluster of 13 molecules shown in Fig. 1.

\begin{tabular}{rrrcccc}
\hline \hline $\mathrm{i}$ & 1 & 2 & 3 & 4 & 5 & 6 \\
\hline$r_{i} / r_{1}$ & 1 & $\sqrt{2}$ & $\sqrt{3}$ & $\sqrt{8 / 3}$ & $\sqrt{11 / 3}$ & 2 \\
$a_{i}$ & 72 & 24 & 36 & 6 & 12 & 6 \\
\hline
\end{tabular}

cluster of $\mathrm{H}_{2}$ molecules whose centers are arranged in an hcp lattice but have otherwise random orientation is given as a convolution of two functions, the one denoting the distribution of separations between lattice points, which we denote by $g_{\text {hcp }}(r)$, and the other representing the molecular structure, i.e., the function $g_{\text {intra }}(r)$ in Eq. (12),

$$
g_{\text {inter }}(r)=\int d^{3} r^{\prime} g_{\text {hcp }}\left(\mathbf{r}^{\prime}\right) g_{\text {intra }}\left(\mathbf{r}-\mathbf{r}^{\prime}\right) .
$$

The correlation of lattice points $g_{\text {hcp }}$ is given by a sum of $\delta$ functions,

$$
n g_{\mathrm{hcp}}(r)=\frac{1}{N} \sum_{i} a_{i} \frac{\delta\left(r-r_{i}\right)}{4 \pi r_{i}^{2}},
$$

where $r_{1}=7.67$ a.u. denotes the nearest-neighbor distance, $r_{2}$ is the second-nearest-neighbor distance, and so on. The coefficients $a_{i}$ represent the number of pairs of lattice points separated by $r_{i}$ in the cluster. [In order to satisfy the normalization condition Eq. (10), each pair is counted twice.] For the tetrahedron, the only separation is $r_{1}$, and $a_{1}=12$. Table $I$ gives the corresponding values of $r_{i}$ and $a_{i}$ for the cluster with 13 molecules.

Figure 2 illustrates the pair-correlation function for a hydrogen cluster consisting of 13 molecules, cf. Fig. 1. Here, the pure hcp structure has been modified due to the convolution with the intramolecular function. Together with the ordered-cluster model is shown the paircorrelation function pertinent to the random-cluster model discussed in Sec. III A, for two different values for

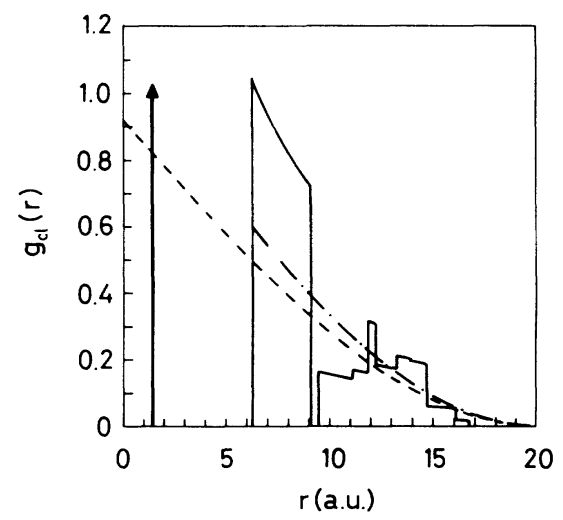

FIG. 2. Various models for the pair-correlation function of atoms in a cluster consisting of 13 molecules. Solid line: hcp structure. Dashed line: random cluster without exclusion volume. Dashed-dotted line: random cluster with exclusion volume according to $r_{\mathrm{ex}}=6.29$ a.u. The thick vertical arrow at $r=r_{\text {mol }}=1.4$ a.u. shows the intramolecular $\delta$ function, Eq. (12). the exclusion radius, $r_{\mathrm{ex}}=0$ and $r_{\mathrm{ex}}=6.29 \mathrm{a} . \mathrm{u}$. The latter value is equal to the one corresponding to the orderedcluster model. Clearly, the curve for the ordered cluster shows a strong nearest-neighbor peak and some structure at larger distances. The curves for the random cluster display quite a different, monotonic behavior, although the pair-correlation function extends up to roughly the same values of $r$ for both the random and the ordered model. All curves have the same normalization, cf. Eq. (10).

\section{CLUSTER STOPPING POWER}

Now we turn to the evaluation of the cluster stopping power Eq. (6) for the models of cluster structure specified above. The separation of intra- and intermolecular effects according to Eq. (11) suggests splitting up the total reduced cluster stopping power $S_{\mathrm{cl}} /\left(N Z^{2}\right)$ into three contributions,

$$
\frac{S_{\mathrm{cl}}}{N Z^{2}}=S_{p}+S_{\text {intra }}+S_{\text {inter }},
$$

where the three terms on the right-hand side denote the respective contributions of independent particles, intraand intermolecular interference. The first of these $S_{p}$ is the stopping power of an isolated proton Eq. (2).

It is clear that, apart from the cluster structure, the behavior of the cluster stopping power will be determined by the dielectric properties of the target medium. The effect of the target properties, modelled by Drude and Mermin dielectric functions, on the cluster stopping has been reported elsewhere [19] for the case of a carbon target. In this article we consider hydrogen stopping in an aluminum target, which is well described by a freeelectron gas, hence one may use Lindhard's dielectric function [31], with the electron density corresponding to the valence-electron density of $\mathrm{Al}$, given by the oneelectron radius $r_{s}=2.07 \mathrm{a} . \mathrm{u}$. This model allows for the separation of individual electron-hole excitations caused by close collisions and collective plasmon excitations due to distant collisions. Plasmons may be excited up to a limiting wave number $k_{c}$, with $k_{c}=0.684 \mathrm{a}$.u. for aluminum.

Regardless of which model is chosen for the paircorrelation, further evaluation of Eq. (6) using Lindhard's dielectric function must proceed numerically. However, a simplification in the description of the target response occurs at cluster velocities that are high compared to the Fermi velocity of the target electrons, where an analytical treatment of cluster interference effects is possible. Therefore, we first present this special case before proceeding with more exact numerical integrations.

\section{A. High-velocity approximation}

For cluster velocities high compared to the Fermi velocity $v \gg v_{F}$, one may describe the electron-gas response by the plasmon-pole approximation [32],

$$
\operatorname{Im}\left(\frac{-1}{\epsilon(k, \omega)}\right)=\frac{\pi \omega_{p}^{2}}{2 \omega_{k}} \delta\left(\omega-\omega_{k}\right),
$$


where $\omega_{p}$ is the plasma frequency and $\omega_{k}$ represents the plasmon dispersion relation

$$
\omega_{k}^{2}=\omega_{p}^{2}+\frac{3}{5} v_{F}^{2} k^{2}+k^{4} / 4 .
$$

This model, applicable in the high-velocity limit, provides a fairly good approximation to the Lindhard dielectric function; it accounts for the collective electron-gas behavior at small $k$ and the free-particle behavior at large $k$, and also includes, although in a rudimentary manner, the Pauli exclusion principle through the appearance of a dispersive term for small $k$, whose value corresponds to a degenerate electron gas with Fermi velocity $v_{F}$. It does not include, however, the Doppler broadening of individual excitations [33] and thus breaks down at low velocities $v \lesssim v_{F}$.

Furthermore, for the sake of analytical convenience we consider here only random clusters with the radius of exclusion $r_{\mathrm{ex}}$ equal to zero [cf. Eq. (16)]. With these simplifications, the evaluation of the stopping power according to Eq. (6) may be carried out analytically and is performed in the Appendix.

For the following discussion it is instructive to study the high-velocity limit of the results in Eqs. (A4) in the Appendix. In this limit, it is $k_{\max } \simeq 2 v$ and $k_{\min } \simeq \omega_{p} / v$. At sufficiently high velocities, $k_{\max }$ is large compared to $k_{\min }$ and the terms in Eqs. (A4b) and (A4c) containing $k_{\max }$ become irrelevant. An expansion for small $k_{\text {min }}$ gives the simple expressions

$$
\begin{aligned}
& S_{\text {intra }} \simeq \frac{\omega_{p}^{2}}{v^{2}} \ln \frac{k_{\text {intra }}}{k_{\text {min }}}, \\
& k_{\text {intra }}=e^{1-\gamma / r_{\text {mol }} \simeq 1.53 / r_{\text {mol }},} \\
& S_{\text {inter }} \simeq(N-2) \frac{\omega_{p}^{2}}{v^{2}} \ln \frac{k_{\text {inter }}}{k_{\text {min }}}, \\
& k_{\text {inter }}=e^{7 / 4-\gamma /\left(2 r_{\mathrm{cl}}\right)} \simeq 1.62 / r_{\mathrm{cl}} .
\end{aligned}
$$

where $\gamma=0.57722$ is Euler's constant. These expressions have a form similar to the proton stopping power $S_{p}$ in Eq. (A4a), with $k_{\max }$ replaced by $k_{\text {intra }}$ for the intramolecular term and by $k_{\text {inter }}$ for the intermolecular term. The two quantities $k_{\text {intra }}$ and $k_{\text {inter }}$ are the respective characteristic wave numbers for the overall interference effect.

It is noted that the $S_{\text {intra }}$ term in Eq. (23) holds for velocities $v \gtrsim \omega_{p} r_{\text {mol }}$, which is essentially the velocity regime $v \gtrsim v_{F}$ of the model used here. $S_{\text {inter }}$, Eq. (24), on the other hand, applies only at much higher velocities $v \gtrsim \omega_{p} r_{\mathrm{cl}}$.

Figure 3 shows the three contributions to the reduced stopping power according to Eq. (20), for a large random cluster of $100 \mathrm{H}_{2}$ molecules impinging on aluminum. There is a threshold velocity, common to all three curves, below which no stopping occurs. This is a well-known characteristic of the plasmon-pole approximation. Above threshold, the term $S_{p}$ goes through a pronounced maximum and then falls off with increasing velocity. The term $S_{\text {intra }}$ shows a similar behavior, but is only about half the magnitude of $S_{p}$. This is easily explained since the characteristic wave number $k_{\text {intra }}$ lies in between $k_{\text {min }}$

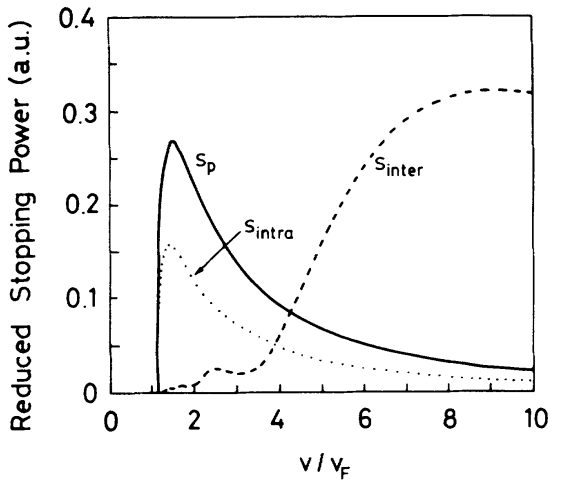

FIG. 3. Contributions $S_{p}, S_{\text {intra }}$, and $S_{\text {inter }}$ to the reduced stopping power of a hydrogen cluster of 100 molecules stopping in aluminum, as a function of $v / v_{F}$. The calculations assume a random cluster with zero distance of exclusion. Target properties are described by a plasmon-pole approximation.

and $k_{\max }$, and thus, roughly speaking, half of all possible excitations will interfere constructively as compared with the individual stopping term $S_{p}$. The intermolecular term $S_{\text {inter }}$, on the other hand, shows quite a different behavior. In the region $v \lesssim 4 v_{F}$, this contribution remains rather small, since possible excitations are restricted to wave numbers that exceed $k_{\text {inter }}$. With increasing velocity, however, excitations with sufficiently small wave numbers, where intermolecular interference is constructive, become accessible. In that velocity region, the intermolecular term dominates because of the prefactor $(N-2)$, i.e., the large number of scatterers involved.

The approximate model, Eq. (24), is useful also to estimate and scale the values of the interference term $S_{\text {inter }}$ for clusters of arbitrary size. Using $k_{\min }=\omega_{p} / v$, the maximum value for the intermolecular interference $S_{\text {inter }}$ becomes $0.64(N-2) / r_{\mathrm{cl}}^{2}$, and occurs at a velocity $v=0.7 \omega_{p} r_{\mathrm{cl}}$. These values agree very well with the results shown in Fig. 3.

\section{B. Full velocity range}

In this section we present results for the cluster stopping power obtained by full numerical evaluation of Eq. (6) using the Lindhard dielectric function. Unlike in the previous section, there will be no restriction on cluster velocity apart from the general assumption of linear dielectric response [31].

First we show in Fig. 4 the influence on $S_{\text {inter }}$ of the distance of exclusion $r_{\mathrm{ex}}$. The system is the same as in Fig. 3, i.e., a random cluster of $100 \mathrm{H}_{2}$ molecules stopping in aluminum. The three curves correspond to different values of $r_{e x}$, respectively, zero, half the nearest-neighbor distance $r_{1}$ of the hcp molecular lattice, and the value $r_{1}-r_{\text {mol }}$ corresponding to the solidhydrogen phase. Note that the curve for $r_{\mathrm{ex}}=0$ looks similar to that of Fig. 3 where the plasmon-pole approximation was used for the dielectric response, and indeed, within the resolution of the figures displayed here, the two results are indistinguishable. The most striking effect of a nonzero distance of exclusion $r_{\mathrm{ex}}$ is the appearance of 


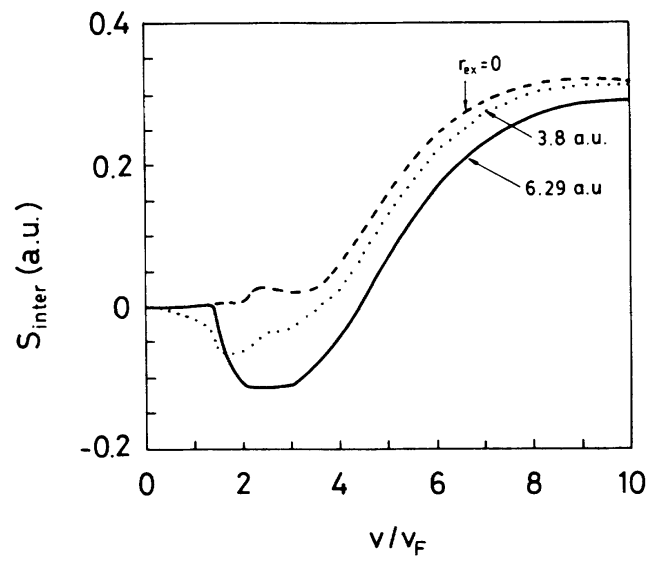

FIG. 4. Intermolecular interference term $S_{\text {inter }}$ for a random hydrogen cluster of 100 molecules stopping in $\mathrm{Al}$, as a function of $v / v_{F}$, for $r_{\mathrm{ex}}=0$ (dashed), 3.8 a.u. (dotted), and 6.29 a.u. (solid line). Target properties are modeled with Lindhard's dielectric function.

a region of negative values of $S_{\text {inter }}$, i.e., destructive intermolecular interference in the immediate vicinity of each molecule. A similar, but smaller, effect has been observed experimentally using diatomic clusters $[6,7]$. A recent experiment using larger clusters [20] shows clearly, similar to the present study, a defect of stopping at low velocities and an excess of stopping at high velocities. Figure 4 shows that the quantitative results will be more sensitive to the short-range order in the cluster, represented here by only one number $r_{\text {ex }}$, in the intermediate range of velocities than for higher velocities.

It is interesting to study the partitioning of energy losses into individual and collective excitations, in particular with respect to the intra- and intermolecular interference. Figure 5 shows all pertinent terms for a random cluster of $13 \mathrm{H}_{2}$ molecules. Consider first the independent-particle contributions due to electron-hole $\left(S_{p}^{e-h}\right)$ and plasmon $\left(S_{p}^{\mathrm{pl}}\right)$ excitation. At low velocities,

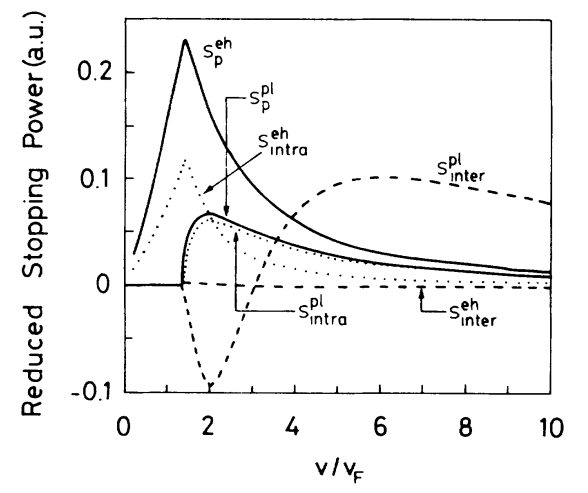

FIG. 5. Various contributions to the stopping power as a function of velocity of a random hydrogen cluster of 13 molecules, with $r_{\mathrm{ex}}=6.29$ a.u. The stopping medium is aluminum, modeled by Lindhard's dielectric function. only the electron-hole mechanism is possible. There is a threshold for plasmon excitation, and for velocities above this threshold both mechanisms give comparable contributions to the stopping. This is known as the partition rule $[22,34]$.

For the intramolecular interference terms $S_{\text {intra }}^{e-h}$ and

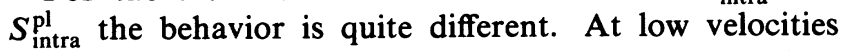
electron-hole excitations give the main contribution, while plasmon excitation is not possible below the threshold velocity. Comparison of the two curves for $S_{p}^{e-h}$ and $S_{\text {intra }}^{e-h}$ shows that intramolecular interference of electronhole excitations is notable (more than $50 \%$ ) at low velocities and is less pronounced at higher velocities. Intramolecular interference of plasmon excitations, on the other hand, gives the major portion at high velocities. Note that the curve for $S_{\text {intra }}^{\text {pl }}$ is strikingly close to the curve for $S_{p}^{\mathrm{pl}}$ : The intramolecular interference of plasmons is constructive and almost complete. Obviously, the wavelengths of plasmons $\lambda \simeq 2 \pi v / \omega_{p}$ are large compared to the interatomic separation within an $\mathrm{H}_{2}$ molecule.

The intermolecular interference terms $S_{\text {inter }}^{e-h}$ and $S_{\text {inter }}^{\mathrm{pl}}$, in turn, show still another behavior. Almost all intermolecular interference is due to plasmon excitation alone, while electron-hole excitations practically do not contribute in this case. Again, this is explained by the pertinent characteristic wave numbers involved: Electron-hole excitations have short wavelengths compared to the extensions of the cluster.

We see in Fig. 5 that the partitioning of energy losses at high velocities due to electron-hole excitations on the one hand, and plasmon excitations on the other, is quite different in the case of cluster stopping compared to proton stopping. Plasmon excitations play a more important role in the case of cluster stopping because of pronounced interference effects, which arise especially at high velocities. At intermediate velocities, the intermolecular interference term due to plasmon excitation is found to be negative, while all other terms are non-negative over the entire velocity range.

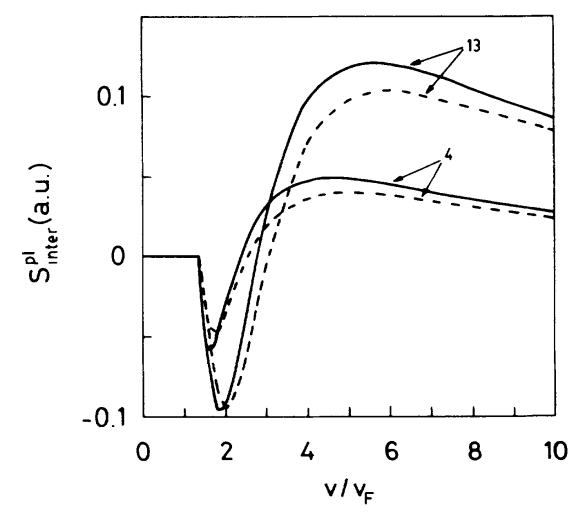

FIG. 6. Intermolecular interference of plasmon excitations for a random (dashed) and an ordered (solid line) hydrogen cluster of 4 and 13 molecules. For the random cluster, it is $r_{\text {ex }}=6.29$ a.u. The stopping medium is aluminum, modeled by a Lindhard dielectric function. 
An important question is the influence of the detailed arrangement of molecules in the cluster on the stopping. Evidently, this will only affect the intermolecular part, and, based on Fig. 4, one would expect the influence to be decreasing with increasing velocity. Figure 6 shows a comparison of intermolecular interference for random versus ordered clusters, as introduced in Sec. III, for two clusters containing 4 and $13 \mathrm{H}_{2}$ molecules. Only the plasmon contribution is displayed, since electron-hole excitations are, in this case, irrelevant, cf. Fig. 5. Very little difference is observed between the two extreme models of random and ordered clusters, having in mind that the pertinent pair-correlation functions differ quite drastically, cf. Fig. 2. Note, however, that this result depends on the choice of the exclusion radius for the random cluster. In Fig. 6, $r_{\mathrm{ex}}$ has been set to 6.29 a.u., corresponding to the value for the ordered cluster. We thus conclude that while short-range order has a definite effect on cluster stopping (see Fig. 4), long-range order in the cluster structure is much less important, at least for the case of random orientation.

In Fig. 7(a) we show the total reduced stopping power for clusters of $1,4,13$, and 100 molecules, together with the proton stopping power as a reference. With increasing cluster size, one observes a gradual change from the familiar single-maximum behavior to a more complex, double-peaked shape, where the first maximum occurs at the proton stopping-power maximum. The second maximum occurs at a somewhat higher velocity that depends on cluster size. From the previous figures it is clear that this feature is caused by intermolecular interference of plasmon excitations. Unlike in Ref. [20], we do not observe a saturation with cluster size of the reduced stopping power.

Finally, it may be of interest to estimate the effects of Coulomb repulsion on cluster stopping. Rather than attempting an elaborate model, we perform here an orderof-magnitude estimate by comparing the previous results with the predictions for an artificially expanded cluster, as one would expect that the cluster will spread and disperse after penetration in dense media. The details of this spreading may be quite complex, and we will confine our considerations to two simplified models, the one corresponding to a homogeneous, uniform expansion of the entire cluster, and the other corresponding to an expansion of the individual molecules only, keeping the relative molecular positions unchanged. The latter model intends to account for the inverse-square law of Coulomb forces. The results in Fig. 7(a) correspond to the original values of the intra- and intermolecular distances in stationary hydrogen clusters, while the results in Figs. 7(b) and 7(c) correspond to expanded clusters. In Fig. 7(b) all intramolecular distances have been enlarged by a factor of 2 , whereas all intermolecular distances are identical to those of Fig. 7(a). The qualitative behavior is not affected by this procedure; in particular, the large enhancement in stopping at high velocities for the cluster of $100 \mathrm{H}_{2}$ molecules is fully reproduced. At velocities near the proton stopping-power maximum, however, interference effects become less pronounced for the expanded cluster.

Figure 7(c) shows the reduced cluster stopping for a
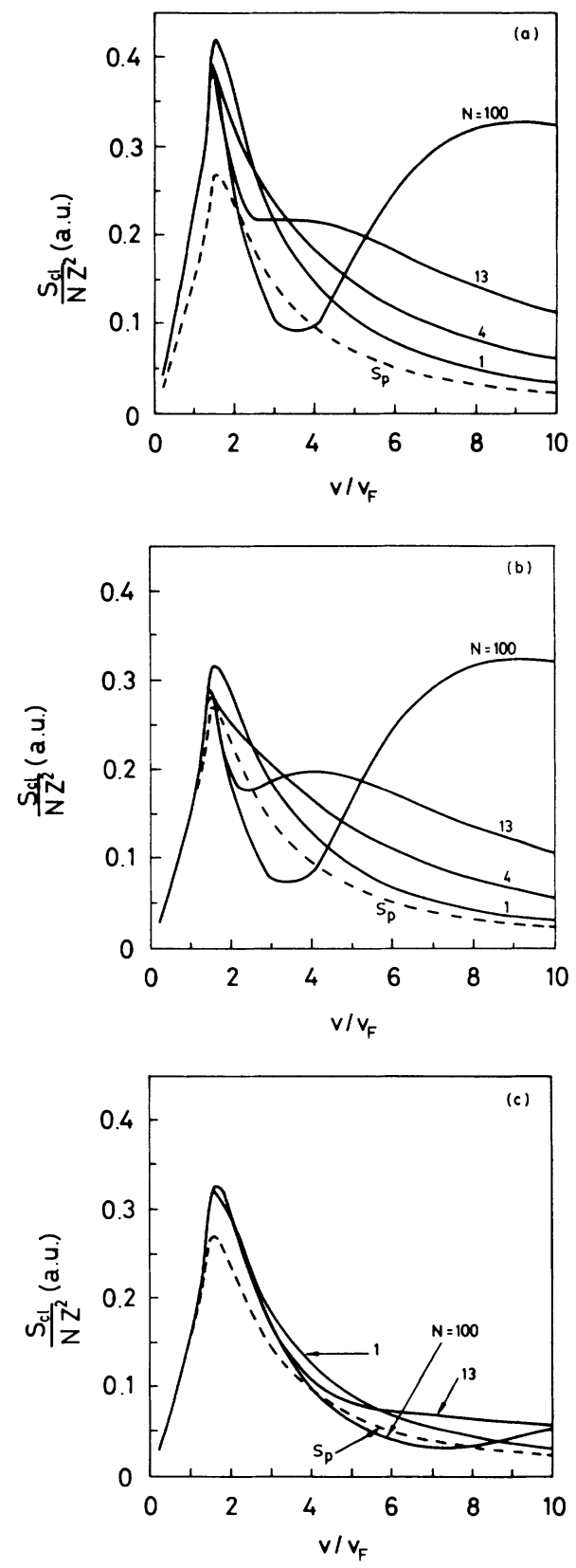

FIG. 7. Total reduced cluster stopping power as a function of velocity for a random hydrogen cluster of $1,4,13$, and $100 \mathrm{~mol}-$ ecules, with $r_{\mathrm{ex}}=6.29$ a.u., together with the proton stopping power as a reference. The stopping medium is aluminum, modeled by Lindhard's dielectric function. The curves (a) show the original-size cluster referred to above; (c) the cluster is expanded homogeneously, i.e., all lengths are stretched by a factor of 2. (b) The intramolecular separations are stretched by a factor of 2, while the intermolecular distances are the same.

uniformly expanded cluster: All intra- and internuclear distances have been enlarged by a factor of 2 . It is observed that the interference effects are much weaker for this expanded cluster. All curves for the reduced stopping power fall close to the proton stopping power $S_{p}$. This is consistent with the scaling argument given at the end of Sec. V. Accordingly, the maximum value for the 
term $S_{\text {inter }}$ is proportional to $1 / r_{\mathrm{cl}}^{2}$, i.e., is reduced by a factor of 4 for the expanded cluster. Also, this maximum occurs at a velocity proportional to $r_{\mathrm{cl}}$, hence the maximum for the cluster of 100 molecules seen at the highvelocity end of Fig. 7(a) is shifted to velocities beyond the range of Fig. 7(c).

\section{CONCLUSIONS}

We have presented calculations of the stopping power of large hydrogen clusters penetrating an aluminum target. Our results concern, on the one hand, the influence of the cluster structure on the stopping power, and on the other, the partitioning of the energy loss among the various possible modes of excitation and interference.

It is found that the detailed spatial distribution of nuclei in the cluster has surprisingly little influence on the stopping power. However, effects of short-range order such as the molecular structure and a distance of exclusion around each atom are clearly visible. Long-range order effects are not observed in a comparison of random versus ordered-cluster stopping powers, at least not for random cluster orientation, as assumed in all our calculations. We conclude that a random spherical cluster mod$\mathrm{el}$, as presented in Sec. III A, may serve as a sufficient model to calculate cluster stopping powers.

Interference effects may be classified into intra- and intermolecular effects, and for an aluminum target they may be subdivided further into electron-hole and plasmon excitations. Electron-hole excitations are of minor importance for intramolecular interference (except at low velocities) and are totally insignificant for intermolecular interference. This is not unexpected since electron-hole excitations may take place within a wide band of wave numbers, thus there occur a whole range of Fourier components which, in part, tend to cancel. Plasmon excitations, on the other hand, exhibit strong interference effects since the associated wavelengths match with the interatomic distances. In the intramolecular case interference is constructive and nearly complete, whereas in the intermolecular case it may be destructive or constructive, depending on velocity. Either way, intermolecular interference due to plasmon excitations is a very pronounced effect for large clusters because of the large number of excitation centers participating. In general, the partitioning of electron-hole and plasmon excitations in the case of a cluster projectile is substantially different from the behavior of a point charge projectile.

The question arises whether the penetration of clusters containing up to 100 molecules may still be treated within the linear-response picture, i.e., first-order perturbation theory. This scheme is a good approximation as long as multiple excitations are negligible. In the cluster frame of reference, this means that target electrons may undergo no more than one collision during their passage of the cluster. A rough estimate shows that the transport mean-free path for electrons moving with $v=1$ a.u. in solid hydrogen of density $0.627 \times 10^{-2}$ a.u. is around 30 a.u. and is increasing very steeply with increasing velocity. From this one would conclude that multiple collisions with notable deflection of an electron penetrating a clus- ter of even $100 \mathrm{H}_{2}$ molecules should be a rare event at velocities $v \gtrsim 1$ a.u., hence validating the linear-response assumption. This simple argument, however, hinges upon the independence of two subsequent collisions (loss of phase) and may thus have to be modified in the present context, particularly in view of the strong interference effects encountered here.

The gross features of the cluster effect on stopping, i.e., a defect of stopping at low velocities and an excess at high velocities, is in qualitative agreement with recent experiments [20].

\section{ACKNOWLEDGMENTS}

Thanks are due to V. Esteve for his skillfull assistance. This collaboration was made possible through financial support to M. Vicanek, sponsored by the Spanish Ministerio de Educación y Ciencia. N.R. Arista received support under the program PROPI (Conselleria d'Educació i Ciència de la Generalitat Valenciana). Partial support from the Dirección General de Investigatión Científica y Técnica (DGICYT) (Projects Nos. PS88-0066 and PS89-0065), and a NATO Grant (No. CRG 901013) is recognized.

\section{APPENDIX}

The plasmon-pole approximation Eq. (21) facilitiates the integration in Eq. (6) over $\omega$,

$$
S_{\mathrm{cl}}=N Z^{2} \frac{\omega_{p}^{2}}{v^{2}} \int_{k_{\min }}^{k_{\max }} \frac{d k}{k} F(k),
$$

with the integration limits $k_{\min }$ and $k_{\max }$ given by the two positive solutions of the equation $\omega_{k}=k v$, cf. Eq. (22),

$k_{\min , \max }^{2}=2\left[\left(v^{2}-\frac{3}{5} v_{F}^{2}\right) \mp \sqrt{\left(v^{2}-\frac{3}{5} v_{F}^{2}\right)^{2}-\omega_{p}^{2}}\right]$.

Then, the integration in Eq. (7) may be performed in closed form for $r_{\mathrm{ex}}=0$, and the structure factor becomes

$$
\begin{aligned}
F(k)=1 & +\frac{\sin \left(k r_{\mathrm{mol}}\right)}{k r_{\mathrm{mol}}} \\
& +(N-2)\left[3 \frac{\sin \left(k r_{\mathrm{cl}}\right)-k r_{\mathrm{cl}} \cos \left(k r_{\mathrm{cl}}\right)}{k^{3} r_{\mathrm{cl}}^{3}}\right)^{2},
\end{aligned}
$$

where the second term on the right-hand side stems from the intramolecular correlation Eq. (12), while the last one is due to the intermolecular part Eq. (16). Note that the latter term is non-negative, hence the intermolecular interference will never be destructive for this particular case of a random cluster with $r_{\mathrm{ex}}=0$. This result is valid independently of the specific dielectric function used.

In view of Eq. (A3), the remaining integration in Eq. (A1) may also be carried out analytically. Splitting the result as in Eq. (20), one gets

$$
S_{p}=\frac{\omega_{p}^{2}}{v^{2}} \ln \frac{k_{\max }}{k_{\min }},
$$


$S_{\text {intra }}=\frac{\omega_{p}^{2}}{v^{2}}\left[\mathrm{Ci}\left(k_{\max } r_{\operatorname{mol}}\right)-\mathrm{Ci}\left(k_{\min } r_{\text {mol }}\right)\right.$

$$
\left.-\frac{\sin \left(k_{\max } r_{\text {mol }}\right)}{k_{\max } r_{\operatorname{mol}}}+\frac{\sin \left(k_{\min } r_{\text {mol }}\right)}{k_{\min } r_{\operatorname{mol}}}\right),
$$

$S_{\text {inter }}=(N-2) \frac{\omega_{p}^{2}}{v^{2}}\left[H\left(2 k_{\max } r_{\mathrm{cl}}\right)-H\left(2 k_{\min } r_{\mathrm{cl}}\right)\right]$.

(A4c)

Here, $\mathrm{Ci}(x)$ denotes the cosine integral [35] and $H(x)$ is defined as

$$
\begin{aligned}
H(x)= & \frac{x^{4}-6 x^{2}+48}{x^{6}} \cos x-\frac{x^{4}-2 x^{2}-48}{x^{5}} \sin x \\
& -\frac{18 x^{2}+48}{x^{6}}+\operatorname{Ci}(x) .
\end{aligned}
$$

An expression similar to the intramolecular term $S_{\text {intra }}$ in Eq. (A4b) has been derived for diclusters in an earlier work $[4,21]$ by two of us.
*Permanent address: Institut für Theoretische Physik, TU, P.O. Box 3329, W-3300 Braunschweig, Germany.

†Permanent address: Instituto Balseiro and Centro Atómico Bariloche, RA- 8400 S.C. Bariloche, Argentina.

[1] J. Remillieux, Nucl. Instrum. Methods 170, 31 (1980).

[2] W. Brandt, A. Ratkowski, and R. H. Ritchie, Phys. Rev. Let. 33, 1329 (1974).

[3] N. R. Arista and V. H. Ponce, J. Phys. C 8, L188 (1975).

[4] N. R. Arista, Phys. Rev. B 18, 1 (1978).

[5] J. W. Tape, W. H. Gibson, J. Remillieux, R. Laubert, and H. E. Wegner, Nucl. Instrum. Methods 132, 75 (1976).

[6] J. C. Eckardt, G. H. Lantschner, N. R. Arista, and R. A. Baragiola, J. Phys. C 11, L851 (1978); J. Steinbeck and K. Dettmann, ibid. 11, 2907 (1978).

[7] M. F. Steuer, D. S. Gemmell, E. P. Kanter, E. A. Johnson, and B. J. Zabransky, Nucl. Instrum. Methods 194, 277 (1982); M. F. Steuer and R. H. Ritchie, ibid. B33, 170 (1988); R. Levi-Setti, K. Lam, and T. R. Fox, ibid. 194, 281 (1982).

[8] D. S. Gemmell, J. Remillieux, J. C. Poizat, M. J. Gaillard, R. E. Holland, and Z. Vager, Phys. Rev. Lett. 34, 1420 (1975); Z. Vager, D. S. Gemmell, and B. J. Zabransky, Phys. Rev. A 14, 638 (1976).

[9] Z. Vager and D. S. Gemmell, Phys. Rev. Lett. 37, 1352 (1976).

[10] D. S. Gemmell, E. P. Kanter, and W. J. Pietsch, Chem. Phys. Lett. 55, 331 (1978); E. P. Kanter, P. J. Cooney, D. S. Gemmell, Z. Vager, W. J. Pietsch, and B. W. Zabransky, Nucl. Instrum. Methods 170, 87 (1980).

[11] D. S. Gemmell, Nucl. Instrum. Methods 191, 425 (1981).

[12] R. J. Beuhler and L. Friedman, Chem. Rev. 86, 521 (1986).

[13] M. Fallavier, J. Kemmler, R. Kirsch, J. C. Poizat, J. Remillieux, and J. P. Thomas, Phys. Rev. Lett. 65, 621 (1990).

[14] R. J. Beuhler, G. Friedlander, and L. Friedman, Phys. Rev. Lett. 63, 1292 (1989).

[15] C. Carraro, B. Q. Chen, S. Schramm, and S. E. Koonin, Phys. Rev. A 42, 1379 (1990).

[16] T. A. Mehlhorn, J. Appl. Phys. 52, 6522 (1981); J. N. Olsen, T. A. Mehlhorn, J. Maenchen, and D. J. Johnson, ibid, 58, 2958 (1985).

[17] C. Deutsch, Ann. Phys. (Paris) 11, 52 (1986).
[18] C. Deutsch, Laser Part. Beams 8, 541 (1990).

[19] I. Abril, M. Vicanek, A. Gras-Marti, and N. R. Arista, Nucl. Instrum. Methods B67, 56 (1992).

[20] E. Ray, R. Kirsch, H. H. Mikkelsen, J. C. Poizat, and J. Remillieux, Nucl. Instrum. Methods 69, 133 (1992).

[21] N. R. Arista and A. Gras-Marti, J. Phys. Condens. Matter 3, 7931 (1991).

[22] J. Lindhard and A. Winther, Mat. Fys. Medd. Dan. Vid. Selsk. 34, 1 (1964).

[23] J. F. Ziegler, J. P. Biersack, and U. Littmark, The Stopping and Range of Ions in Solids (Pergamon, New York, 1985) p. 5.

[24] N. R. Arista and A. Gras-Marti, in Interaction of Charged Particles with Solids and Surfaces, Vol. 271 of NATO Advanced Study Institute, Series B: Physics, edited by A. Gras-Marti, H. M. Urbassek, N. R. Arista, and F. Flores (Plenum, New York, 1991).

[25] J. M. Ziman, Models of Disorder (Cambridge University Press, Cambridge, 1979).

[26] M. V. Rama Krishna and K. B. Whaley, Z. Phys. D 20, 223 (1991).

[27] M. Chevallier, N. V. de Castro Faria, B. Farizon-Mazuy, M. J. Gaillard, J. C. Poizat, and J. Remillieux, J. Phys. (Paris) 50, c2-189 (1989).

[28] M. P. Iñiguez, M. J. Lopez, J. A. Alonso, and J. M. Soler, Z. Phys. D 11, 163 (1989); A. Mañanes, M. P. Iniguez, M. J. Lopez, and J. A. Alonso, Phys. Rev. B 42, 5000 (1990); U. Lammers, G. Borstel, A. Mañanes, and J. A. Alonso, Z. Phys. D 17, 203 (1990).

[29] V. V. Goldman, J. Low Temp. Phys. 36, 521 (1979).

[30] I. F. Silvera, Rev. Mod. Phys. 52, 393 (1980).

[31] J. Lindhard, Mat. Fys. Medd. Dan. Vid. Selsk. 28, 1 (1954).

[32] L. Hedin and S. Lundqvist, Solid State Phys. 23, 1 (1969); A. W. Overhauser, Phys. Rev. 83, 1888 (1971).

[33] D. Pines, Elementary Excitations in Solids (Benjamin, New York, 1964).

[34] W. Brandt and J. Reinheimer, Phys. Rev. B 2, 3104 (1970).

[35] Handbook of Mathematical Functions, edited by $\mathbf{M}$. Abramowitz and I. S. Stegun (Dover, New York, 1965). 